\title{
The experimental determination of surface tension of supercooled water
}

\author{
Radim Mareš $^{1, a}$ and Jana Kalová ${ }^{2}$ \\ ${ }^{1}$ University of West Bohemia in Pilsen, Faculty of Mechanical Engineering, Czech Republic \\ ${ }^{2}$ University of South Bohemia in České Budějovice, Faculty of Science, Czech Republic
}

\begin{abstract}
The surface tension of supercooled demineralized water in liquid state was measured using the elevation capillary method. Glass capillaries with an inner diameter from $0.35 \mathrm{~mm}$ to $0.20 \mathrm{~mm}$ were taken. The measurement was carried out from the ambient temperature down to $-12{ }^{\circ} \mathrm{C}$ at normal pressure. The measured values of the surface increasing tension at decreasing temperature were compared with published experimental data and with the current IAPWS equation.
\end{abstract}

\section{Introduction}

It is very difficult to get experimental data in the supercooled water region. Measurements of thermophysical properties of water are restricted to temperatures above approximately $233 \mathrm{~K}$, which is the limit of homogenous nucleation. Supercooled water exhibits anomalous behavior and a thermodynamically consistent view on its properties (of the heat capacity, the isothermal compressibility, and the thermal expansivity) is still sought [1].

The surface tension of water is a property important for chemical and biological research or for exploration of atmospheric phenomena. For instance, the surface tension at the interface between liquid and ice influences the formation of ice on aircraft. The surface tension between the vapor and liquid phase is important for modeling of precipitation formed in clouds. Surface tension influences the formation of liquid - water droplets from air saturated with water vapor. The condensation process occurs very often at temperatures below $0{ }^{\circ} \mathrm{C}$ in the atmosphere and in flowing gas streams that are subject to adiabatic cooling.

Anomalous behavior of many thermophysical properties in the supercooled region caused a great attention was paid to the research of the second inflection point, as discussed by Gittens G. J. [2]. However, its existence has neither been confirmed nor denied so far. For temperatures above $0{ }^{\circ} \mathrm{C}$, the inflection point of the temperature dependence of the surface tension is either very weak or does not exist. If we suppose the IAPWS evaluated data to be the best existing data for surface tension above $0{ }^{\circ} \mathrm{C}$, the function of temperature

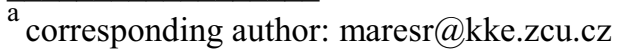

dependence is concave down in this region. That is why the data below $0{ }^{\circ} \mathrm{C}$ are necessary for a test of the existence of the second inflection point.

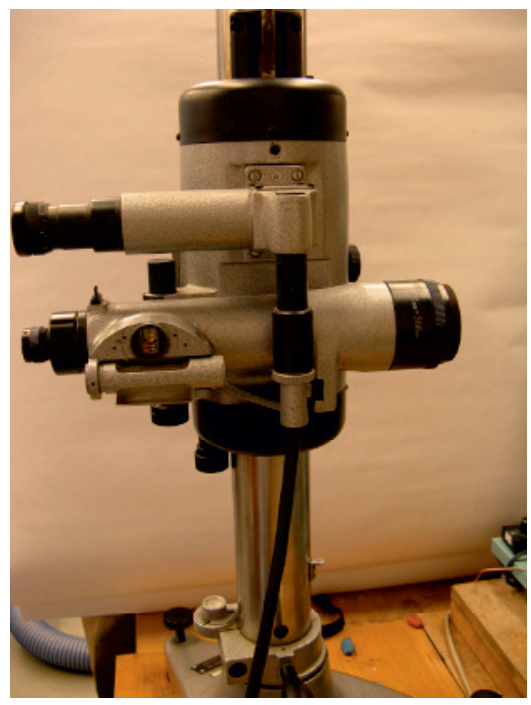

Figure 1. Lomo KM-6 cathetometer

\section{Description of the measuring equipment}

A spherical cap meniscus can be assumed inside capillaries of a small diameter. The change of the demineralized water meniscus position, depending on temperature, was measured using the Lomo KM-6 cathetometer (displayed in figure1) with accuracy of 0.01 $\mathrm{mm}$. Pt100 temperature sensors were connected to a HP 34970A switchboard linked to a computer. All temperature sensors were calibrated with a Pt 100 
standard in the whole temperature range used in the measurement.

Alcohol was used as a cooling medium in the Lauda Proline RP845 thermostat (displayed in figure 2) and was taken by a circulating pump into the piping in the heat exchanger (displayed in figure 3 ).

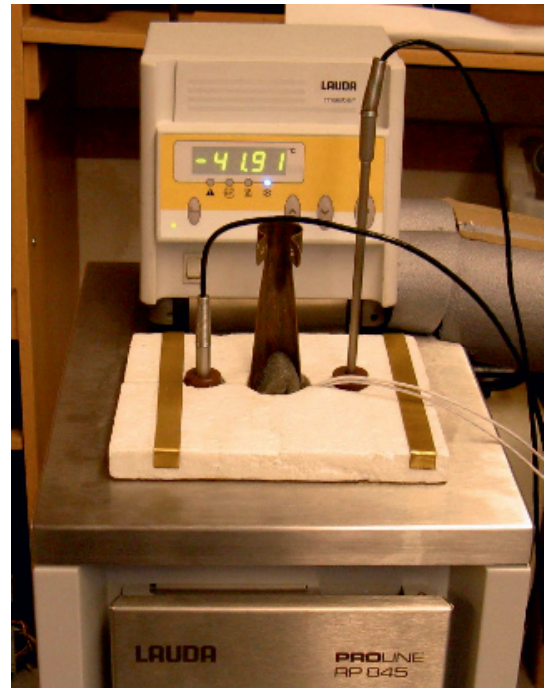

Figure 2. Lauda Proline RP845 thermostat

The helix shaped pipeline is surrounded with a gaseous medium (dried air or nitrogen) which keeps the measuring chamber (displayed in figure 4 ) at the required temperature. The cooling medium should be mixed up in front of the measuring chamber in order to assure its homogeneous temperature in the chamber.

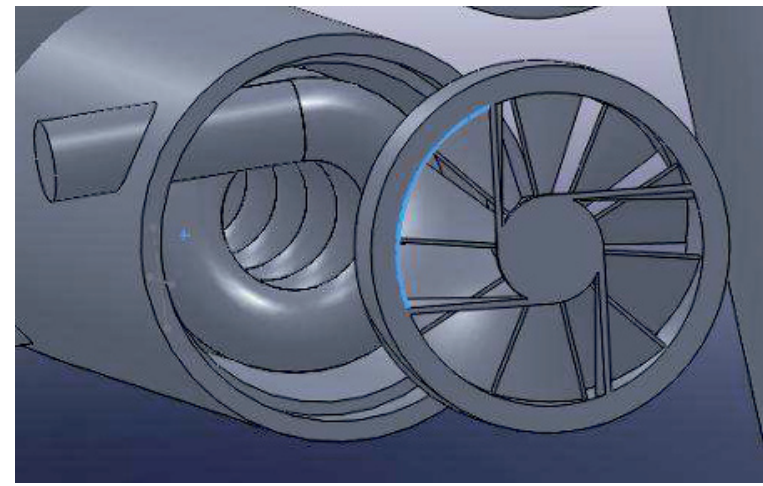

Figure 3. Heat exchanger

Values of the surface tension were calculated from the measured meniscus elevation at different temperatures of water in the capillary.

\section{Existing experimental data}

For the above mentioned reasons, the research of the temperature dependence was shifted to the region of supercooled water. Two independent basic measurements for the surface tension exist in this region [3, 4]. These data show a slightly different trend. Data [3] are well approximated by extrapolating of the international IAPWS equation for the surface tension [1], outside the range validity for temperatures below $0{ }^{\circ} \mathrm{C}$. The data [4] are quite well confirmed by calculations based on molecular dynamic methods [5]. For these reasons, it was appropriate to take additional independent measurements of the temperature dependence of the surface tension.

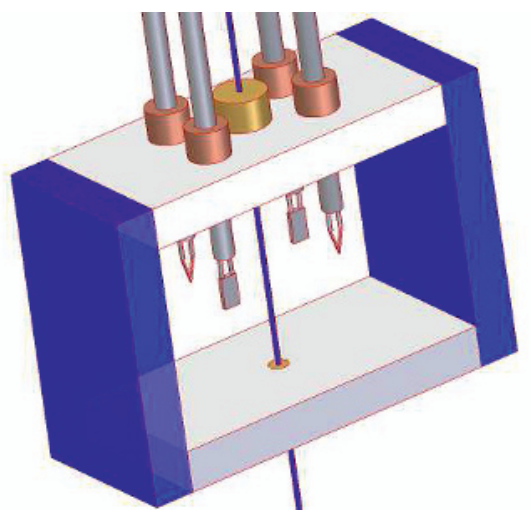

Figure 4. Measuring chamber

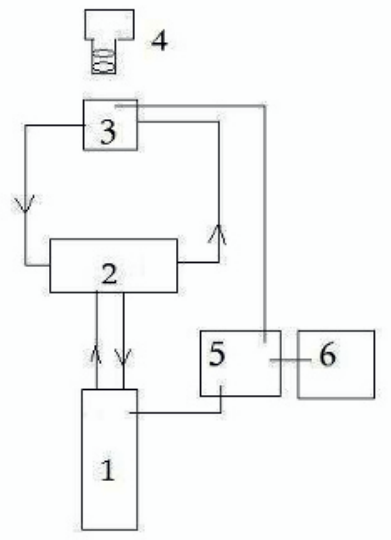

Fig. 5. Scheme of the device: 1 thermostat, 2 heat exchanger, 3 measuring chamber, 4 cathetometer, 5 switchboard, 6 PC

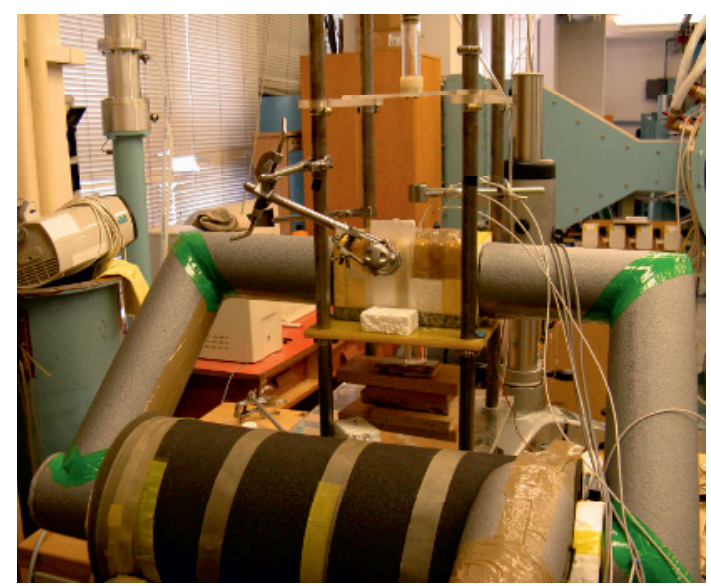

Figure 6. Measuring device organization 


\section{Processing of measured values}

If the column of a liquid of density $\rho$ in a capillary with the inner diameter $d$ goes up over the surface to a height $h$, and if the contact angle between the liquid and the container is $\theta$, then from the equilibrium between the gravity force of the liquid in the capillary and the surface tension force with the incline $\sigma$, this relation for the surface tension results:

$$
\sigma=\frac{\rho g d}{4 \cos \theta} h
$$

Thus, this equation for the surface tension follows:

$$
\sigma=\sigma_{0}+\frac{\rho g d}{4 \cos \theta}\left(h-h_{0}\right)
$$

The temperature $t_{0}=0{ }^{\circ} \mathrm{C}$, the corresponding surface tension $\sigma_{0}$, and the height $h_{0}$ of a column of a liquid was chosen as a reference state.

Water in the capillary between the vessel with water and the measuring chamber of the height $h_{a}$ has ambient temperature and density $\rho_{a}$. Water in the capillary in the measuring chamber of the height $h_{c}$ has temperature of the cooling gaseous medium and density $\rho_{c}$. Therefore the hydrostatic pressure from equation (1)

$$
p_{s}=\rho g h
$$

should be replaced by the equation

$$
p_{s}=\left(\rho_{a} h_{a}+\rho_{c} h_{c}\right) g
$$

The surface subsidence of water in the vessel due to elevation of water column in the capillary is quite negligible and is lower than the display resolution of the cathetometer. Therefore the height $h_{a}$ could be considered a constant value. Subsequently the increment of the height increment in the equation (2)

$$
\Delta h=h-h_{0}
$$

could be replaced by

$$
\Delta h_{c}=h_{c}-h_{c 0} \text {, }
$$

where the height $h_{c 0}$ of a column of a liquid was chosen as a reference state.

Consequently, the equation (2) could be turned into

$$
\sigma=\sigma_{0}+\frac{\rho g d}{4 \cos \theta}\left(h_{c}-h_{c 0}\right) .
$$

\section{Measurement results}

The data gained by the authors of this article (figures 7, 9) correspond rather with the values by Floriano M. A. and Angell C. A. [3]. They reach higher values than data by Trinh E. H. and Ohsaka K. [4], mentioned by Holten et al. [1], but lower than Hacker P. T. [5]. These data have very smooth behavior and can be very accurately approximated using a polynomial of the third degree. Data by Floriano and Angell [3] oscillate significantly (figures 8, 9).

The International Association for the Properties of Water and Steam (IAPWS) has issued the Release on the Surface Tension of Ordinary Water Substance in 1994 [6]. For temperatures between $0.01^{\circ} \mathrm{C}$ and $374{ }^{\circ} \mathrm{C}$, this document contains critically evaluated data of the surface tension $\sigma$, with estimated values of uncertainty $\Delta \sigma$. A very elegant interpolating equation was recommended in the aforesaid IAPWS release in 1994:

$$
\sigma=B \tau^{\mu}(1+b \tau)
$$

where $B=235.8 \mathrm{~m} \mathrm{~N} \cdot \mathrm{m}^{-1}, \quad \tau=1-\frac{T}{T_{c}}$, $\mu=1.256, b=-0.625, T_{c}=647.096 \mathrm{~K}$.

A range of validity of the equation (1) is between the triple point and critical temperature $647.096 \mathrm{~K}$.

It seems very reasonable to apply an extrapolation of the international IAPWS equation outside the range of its validity, below $0{ }^{\circ} \mathrm{C}$.

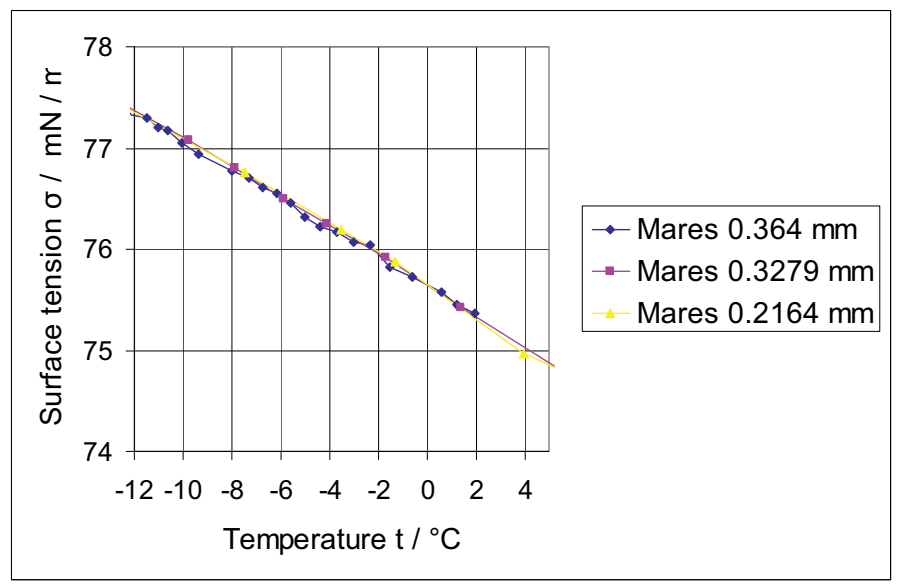

Figure 7. Measurements of Mareš and Kalová

\section{Conclusions}

According to the equation (2), values of the surface tension $\sigma$ were calculated from the measured values 
$h$ at different temperatures of water in the vicinity of the meniscus in the capillary.

The data gained by the authors of this article correspond rather with the values of Floriano and Angell [3], reach higher values than the data of Trinh [4], but the data are lower than those of Hacker [5]. These data have very smooth behavior and can be very accurately approximated using a polynomial of the third degree. Data of Floriano and Angell [3] significantly oscillate. It seems very reasonable to apply an extrapolation of the international IAPWS equation [6] outside the range of its validity, below $0{ }^{\circ} \mathrm{C}$. This equation does not show any inflection point in the field of negative temperatures [7 9].

New experimental data for the surface tension of supercooled water confirm the validity of the extrapolation of the IAPWS equation in the temperature interval from $-12{ }^{\circ} \mathrm{C}$ to $374{ }^{\circ} \mathrm{C}$.

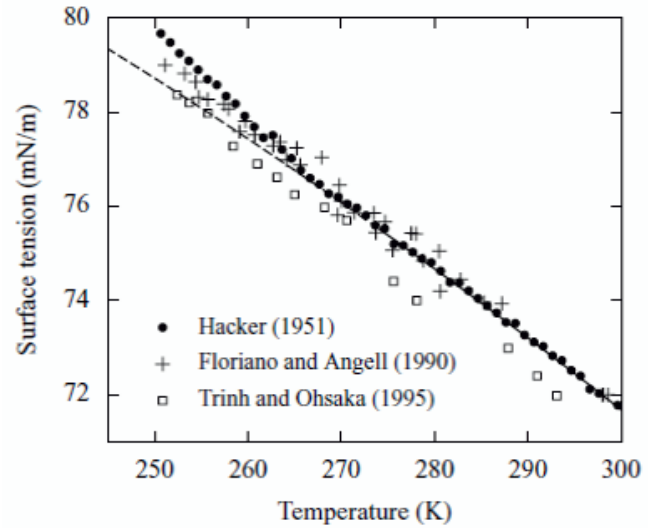

Figure 8. Experimental data Trinh and Ohsaka vs. Hacker, Floriano and Angell, taken from [4]

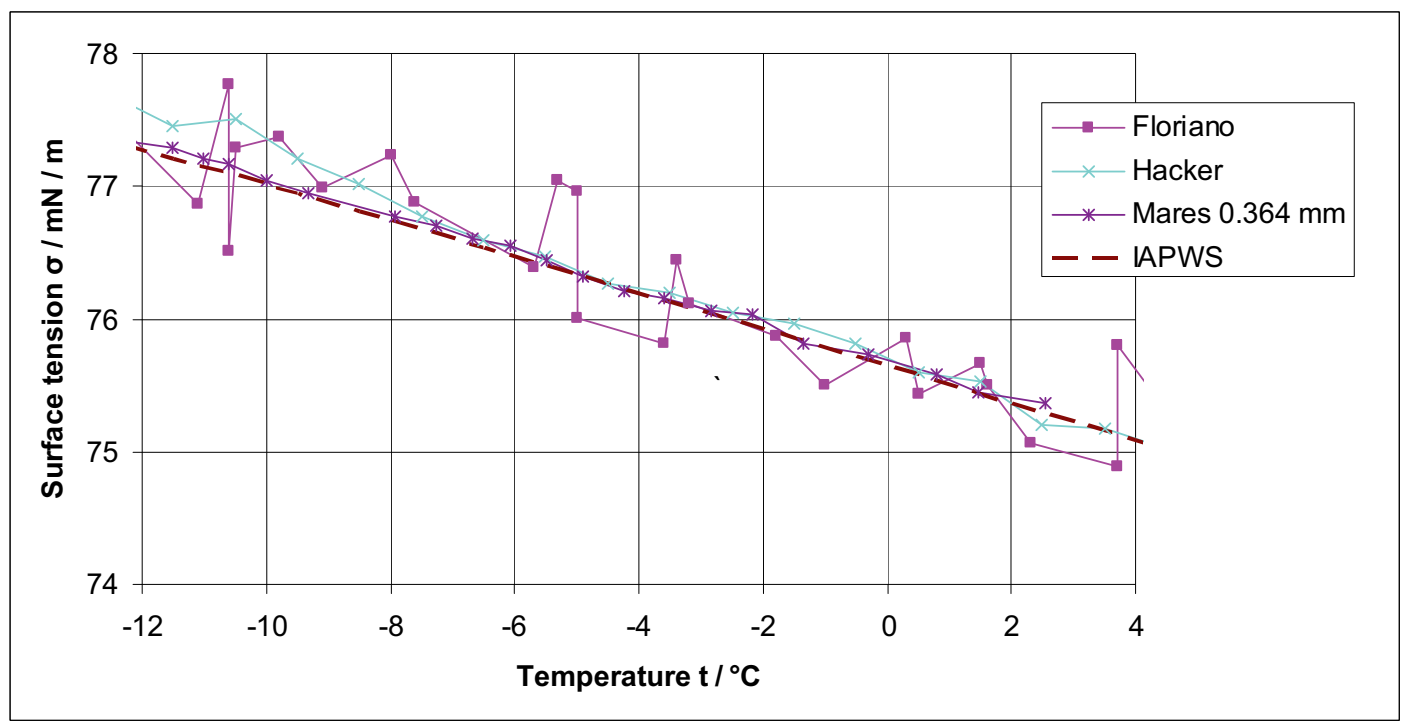

Figure 9. Comparison of experimental data: Floriano and Angell [2], Hacker [5], extrapolated IAPWS equation [6] (dashed line) and results of measurements of Mareš and Kalová with capillary 0.364

Acknowledgment The research was supported by the GA of the Czech Academy of Sciences under the Grant IAA200760905 ,Thermophysical properties of water in unexplored, technologically significant regions" and by the Czech Science Foundation, Grant P101-13-20467P. The authors have benefited from valuable advice and assistance with the head of the Department of Thermodynamics and the head of the Laboratory of Phase Transitions of the Institute of Thermomechanics AS CR, Ing. Jan Hrubý.

\section{References}

1. V. Holten, C. E. Bertrand, M. A. Anisimov, J. V. Sengers, J.Chem.Phys., 136, 094507 (2012)
2. G. J. Gittens, J. Colloid and Interface Sci, 30, 3 (1969)

3. M. A. Floriano, C. A. Angell, J. Phys. Chem., 94, 4199 (1990)

4. E. H. Trinh, K. Ohsaka, K., IJT, 16, 2 (1995)

5. P. T. Hacker, National Advisory Committee for Aeronautics, Technical Note 2510, 1-20 (1951)

6. IAPWS Release on Surface Tension of Ordinary Water Substance, September 1994, Accessed on 15 August 2012 (http://www.iapws.org)

7. J. Kalová, R. Mareš, IJT, 33, 992 (2012)

8. R. Mareš, J. Kalová, 31. setkání kateder mechaniky tekutin a termomechaniky, Mikulov, 145-148 (2012)

9. V. Vinš, J. Hrubý, J. Hykl, J Blaha, B. Šmíd, EPJ Web Conf. 45, 01094 (2013) 